\title{
UNICITY OF THE EXTREMUM PROBLEMS IN $H^{1}\left(U^{n}\right)$
}

\section{Kôzô YABUTA ${ }^{1}$}

Abstract. In 1958 de Leeuw and Rudin have given a sufficient condition for a function in $H^{1}(U)$ to be a unique solution of the extremum problem. We give in this paper a stronger sufficient condition (Theorem 1) which holds also in $n$-dimension. Our Theorem 1 fills up considerably the gap of de Leeuw-Rudin's result. We give also another proof of Neuwirth-Newman's theorem and its $n$-dimensional generalization.

1. Let $S$ be the unit ball of the Hardy class $H^{1}\left(U^{n}\right)$ in the unit polydisc $U^{n}$. If $\phi$ is a bounded measurable function on $T^{n}$ : the distinguished boundary of $U^{n}$, we shall denote by $T_{\phi}$ the bounded linear functional defined on $H^{1}\left(U^{n}\right)$ by

$$
T_{\phi}(f)=\int_{T^{n}} f^{*}(w) \phi(w) d m_{n}(w),
$$

where $m_{n}$ denotes the normalized Haar measure on $T^{n}$, and $f^{*}(w)$ $=\lim _{r \rightarrow 1} f(r w)$ if it exists. The norm of $T_{\phi}$ is

$$
\left\|T_{\phi}\right\|=\sup _{f \in S}\left|T_{\phi}(f)\right|,
$$

and we let $S_{\phi}$ denote the set of all $f \in S$ for which $T_{\phi}(f)=\left\|T_{\phi}\right\|$. The set $S_{\phi}$ is the set of solutions of the extremum problem. Necessarily $f \in S_{\phi}$ must be of norm 1. Here is a lemma which can be obtained in a similar way in $[1]$.

Lemma 1. $f$ and $g$ belong to the same $S_{\phi}$ if and only if

$$
\arg f^{*}(w)=\arg g^{*}(w) \quad \text { a.e. } w \in T^{n}
$$

and $\|f\|=\|g\|=1$.

By this lemma, if $f \in H^{1}\left(U^{n}\right)$ and $\|f\|=1, f$ belongs to one and only one $S_{\phi}=S_{\left|f^{*}\right| / f^{*}}$, which we denote by $S^{f}$.

Now we state

ThEOREM 1. (a) If $f \in H^{1}\left(U^{n}\right),\|f\|=1$, f is outer, and $1 / f^{*} \in L^{1}\left(T^{n}\right)$, then $S^{\prime}$ consists of $f$ alone.

Received by the editors December 19, 1969 and, in revised form, January 17, 1970.

AMS 1970 subject classifications. Primary 32A99; Secondary 30A78.

Key words and phrases. Hardy class, polydisc, extremum problem, extreme point, outer function, inner function, Poisson integral, homogeneous expansion.

1 In this note we use systematically the notations of Rudin's lecture note [3]. 
(b) If $S^{f}$ consists of $f$ alone, then $f$ is an extreme point of $S$ and $f(z) /(1-u(z))^{2}$ fails to be in $H^{1}\left(U^{n}\right)$ for every inner function $u(z)$.

THEOREM 2. If $f \in H^{1}\left(U^{n}\right),\|f\|=1, f$ is outer, and $1 / f^{*} \in L^{p}\left(T^{n}\right)$ $\left(\frac{1}{2} \leqq p<1\right)$, then $S^{f}-\{f\}$ contains no $H^{q}\left(U^{n}\right)(1 / p+1 / q=2)$ function.

THEOREM 3. $S^{\prime}$ consists of $f$ alone if and only if $\|f+g\|<2$ for every $g \in S, g \neq f$.

As a corollary to the proof of the above theorems we can give another proof of Neuwirth and Newman's theorem [2] and its $n$ dimensional generalization.

THEOREM 4. If $f \in H^{1 / 2}\left(U^{n}\right)$ and $f^{*}(w) \geqq 0$ a.e. on $T^{n}$, then $f$ is a constant.

2. The key tools in proving the above theorems are the following lemmas. Lemma 2 is an elemental property of $L^{1}$ and Lemma 3 is given essentially in [1] for $H^{1}(U)$.

Lemma 2. If $f, g \in L^{1}\left(T^{n}\right)$ and $\|f\|=\|g\|=\|(f+g) / 2\|$, then $\arg f$ $=\arg g$ a.e. on $T^{n}$.

Lemma 3. If $f \in H^{p}(U)(p \geqq 1)$, then there exists $g \in H^{p}(U)$ such that

(1) $\|f+g\|_{1}=\|f-g\|_{1}=\|f\|_{1}$ and consequently

$\arg \left(f^{*}(w)+g^{*}(w)\right)=\arg \left(f^{*}(w)-g^{*}(w)\right)=\arg f^{*}(w)$ a.e. on $T$.

(2) $f \pm g$ are outer.

Proof. Let $f=M_{f} Q_{f}$ be the inner-outer factorization of $f$. Clearly there is a real $\alpha$ such that

$$
\int_{T}\left|f^{*}(w)\right| \operatorname{Re}\left[e^{i \alpha} M_{f}^{*}(w)\right] d m_{1}(w)=0 .
$$

Put

$$
u(z)=e^{i \alpha} M_{f}(z), \quad z \in U,
$$

and

$$
g(z)=\frac{1}{2} e^{-i \alpha} Q_{f}(z)\left(1+u^{2}(z)\right), \quad z \in U .
$$

Since $Q_{f}$ is in $H^{p}(U)$, we have $g \in H^{p}(U)$ and

$$
\left|f^{*}(w) \pm g^{*}(w)\right|=\left|f^{*}(w)\right|\left(1 \pm \operatorname{Re}\left[u^{*}(w)\right]\right) \text { a.e. on } T \text {. }
$$

By (2.1) we have, therefore, $\|f+g\|_{1}=\|f-g\|_{1}=\|f\|_{1}$.

Since $u(z)$ is inner, we have $\operatorname{Re}(1 \pm u(z))>0(z \in U)$, and con- 
sequently $1 \pm u$ are outer functions by Lemma 1.4 of [1]. Thus, since $f \pm g= \pm \frac{1}{2} e^{-i \alpha} Q_{f}(1 \pm u)^{2}, f \pm g$ are outer functions.

LEMMA 4. If $f$ is an outer function in $H^{p}(U)\left(\frac{1}{2} \leqq p \leqq 1\right), h \in H^{q}(U)$ $(1 / p+1 / q=2)$, and if $f^{*} h^{*} \geqq 0$ a.e. on $T$, then $f h$ is a constant.

Proof. As $\frac{1}{2} \leqq p \leqq 1$ and $1 / p+1 / q=2$, we have $1 \leqq q \leqq \infty$. Thus by Lemma 3 there is $g \in H^{q}(U)$ such that $h \pm g$ are outer and $\arg \left(h^{*} \pm g^{*}\right)=\arg h^{*}$ a.e. on $T$. As $f$ and $h \pm g$ are outer, they have no zeros in $U$. Hence $\sqrt{ } f \in H^{2 p}(U)^{2}$ and $\sqrt{ }(h \pm g) \in H^{2 q}(U)$, which implies that $\sqrt{ } f \sqrt{ }(h \pm g) \in H^{1}(U)$. Since $f^{*} h^{*} \geqq 0$, and $\arg \left(h^{*} \pm g^{*}\right)=\arg h^{*}$ a.e. on $T$, we have $f^{*}\left(h^{*} \pm g^{*}\right) \geqq 0$ a.e. on $T$, which implies that $(\sqrt{ } f \sqrt{ }(h \pm g))^{*}$ are real a.e. on $T$. These two facts show that $\sqrt{ } f$ $\sqrt{ }(h \pm g)$ are constants since every $H^{1}(U)$ function can be represented by the Poisson integral of its boundary function. This shows clearly that $f h$ is constant.

3. Proofs of Theorem 1(a) and Theorem 2. Since Theorem 1(a) is a consequence of Theorem 2 it is sufficient to prove Theorem 2 . Since $f$ is outer, $1 / f \in N_{*}\left(U^{n}\right)$, so $1 / f^{*} \in L^{p}\left(T^{n}\right)$ implies that $1 / f$ $\in H^{p}\left(U^{n}\right)$. Hence for almost all $w \in T^{n}, f_{w}(\lambda) \in H^{1}(U)$ and $1 / f_{w}(\lambda)$ $\in H^{p}(U)$, where $f_{w}(\lambda)=f(\lambda w)$ for $\lambda \in U$, we $T^{n}$. Let $g \in S^{\prime} \cap H^{q}\left(U^{n}\right)$, then by Lemma 1 , for almost all $w \in T^{n}$, we have $g_{w}^{*}\left(e^{i \theta}\right) / f_{w}^{*}\left(e^{i \theta}\right) \geqq 0$ a.e. on $T$. Thus remarking that $f, 1 / f \in N_{*}(U)$ implies that $f$ is outer, we see, by Lemma 4 , that almost every $g_{w} / f_{w}(\lambda)$ is a constant $a_{w}$. But the $\operatorname{discs}\{\lambda w ;|\lambda|<1\}$ intersect at $(0, \cdots, 0)$, so $g_{w} / f_{w}(\lambda)=g / f(0)$ for almost all $w \in T^{n}$. Expanding $g / f$ homogeneously, we have $g / f=g / f(0)$. Since $\|g\|=\|f\|=1, g / f$ must be 1 .

4. The proof of Theorem 1 (b) follows along the same lines as that of Theorem $8(\mathrm{~b})$ of [1]. Theorem 3 is a variant of Lemma 1 by Lemma 2.

5. Proof of Theorem 4. For the case $n=1$, we have $f=M_{f} Q_{f}$, where $M_{f}$ is inner and $Q_{f}$ is outer and is in $H^{1 / 2}(U)$. Thus by Lemma 4, the fact that $f^{*} \geqq 0$ a.e. on $T$ implies that $f$ is a constant. For general $n$, we have that $f_{w}(\lambda) \in H^{1 / 2}(U)$ and $f_{w}^{*}\left(e^{i \theta}\right) \geqq 0$ a.e. on $T$, for almost all $w \in T^{n}$. Hence by the above discussion it follows that $f_{w}(\lambda)=a_{w}$ for almost all $w \in T^{n}$. The rest of the proof follows along the same lines as that of Theorem 1 (a).

REMARK 1. Theorem 1 fills up the gap of Theorem 8 of [1] considerably, and is, in a sense, sharp. That is, if the assumption $1 / f^{*} \in L^{1}$ is replaced by $1 / f^{*} \in L^{p}(0<p<1),\left(1-z_{1}\right)^{\alpha} /\left\|\left(1-z_{1}\right)^{\alpha}\right\|_{1}(1<\alpha<1 / p)$

2 The branch of $\sqrt{ }$ is such that $\sqrt{ } 1=1$. 
gives an example in which $S^{\prime}$ contains at least two functions, by Theorem 1 (b), where $z=\left(z_{1}, \cdots, z_{n}\right)$.

REMARK 2. The conditions of Theorem 1(a) are equivalent to the condition that both $f$ and $1 / f$ belong to $H^{1}\left(U^{n}\right)$.

Remark 3. We can also prove Theorem 1(a) by using Neuwirth and Newman's theorem, but we dared to show it without use of their theorem. And also after giving the proof of Theorem 4, we can give Theorems 1 and 2 as corollaries of Theorem 4 . But we consider that Lemma 4 is certainly useful.

\section{REFERENCES}

1. K. de Leeuw and W. Rudin, Extreme points and extremum problems in $H_{1}$, Pacific J. Math. 8 (1958), 467-485. MR 20 \#5426.

2. J. Neuwirth and D. J. Newman, Positive $H^{1 / 2}$ functions are constants, Proc. Amer. Math. Soc. 18 (1967), 958.

3. W. Rudin, Function theory in polydiscs, Benjamin, New York, 1969.

Mathematical Institute, Tôhoru University, Sendai, Japan 